\title{
Bifurcations and crises in a shape memory oscillator
}

\author{
Luciano G. Machado ${ }^{\mathrm{a}}$, Marcelo A. Savi ${ }^{\mathrm{b}, *}$ and Pedro M.C.L. Pacheco ${ }^{\mathrm{c}}$ \\ ${ }^{a}$ Instituto Militar de Engenharia, Department of Mechanical and Materials Engineering, 22.290.270, Rio de \\ Janeiro, RJ, Brazil \\ ${ }^{\mathrm{b}}$ Universidade Federal do Rio de Janeiro, COPPE, Department of Mechanical Engineering, 21.945.970, Rio de \\ Janeiro, RJ, Brazil, Caixa Postal 68.503 \\ E-mail:savi@ufrj.br \\ ${ }^{\mathrm{c}}$ CEFET/RJ, Department of Mechanical Engineering, 20.271.110, Rio de Janeiro, RJ, Brazil \\ E-mail: calas@cefet-rj.br
}

Received 5 May 2003

Accepted 24 September 2003

\begin{abstract}
The remarkable properties of shape memory alloys have been motivating the interest in applications in different areas varying from biomedical to aerospace hardware. The dynamical response of systems composed by shape memory actuators presents nonlinear characteristics and a very rich behavior, showing periodic, quasi-periodic and chaotic responses. This contribution analyses some aspects related to bifurcation phenomenon in a shape memory oscillator where the restitution force is described by a polynomial constitutive model. The term bifurcation is used to describe qualitative changes that occur in the orbit structure of a system, as a consequence of parameter changes, being related to chaos. Numerical simulations show that the response of the shape memory oscillator presents period doubling cascades, direct and reverse, and crises.
\end{abstract}

Keywords: Shape memory alloys, chaos, bifurcation, crisis, nonlinear dynamics

\section{Introduction}

Shape memory effect and pseudoelasticity are thermoelastic phenomena, related to martensitic transformation, presented by shape memory alloys (SMAs). The mechanism behind SMAs remarkable behavior is related to martensitic phase transformation that the alloy undergoes when subjected to stress and/or temperature changes. Essentially, there are two basic crystalline states in SMAs: austenite and martensite. Martensitic transformation is a non-diffusive process, which happens due to differences between involved constituents [34]. In brief, it is possible to say that

\footnotetext{
*Corresponding author.
}

martensitic transformations are time independent and temperature dependent.

$\mathrm{Ni}-\mathrm{Ti}, \mathrm{Cu}-\mathrm{Zn}, \mathrm{Mg}-\mathrm{Cu}, \mathrm{Fe}-\mathrm{Mn}-\mathrm{Si}$ and $\mathrm{Cr}-\mathrm{Ni}$ are some SMAs that have been motivating several applications in different areas. Self-actuating fasteners [3,12,13,33], thermally actuator switches and several bioengineering devices are some examples of these applications [6, 14-16]. Aerospace technology is also using SMAs to solve important problems, in particular those concerning with space savings achieved by self-erectable structures, stabilizing mechanisms, non-explosive release devices and other possibilities [5,19]. Micromanipulators and robotics actuators have been built employing SMAs properties to mimic the smooth motions of human muscles [8,10,22,35]. Moreover, SMAs are being used as actuators for vibration and buckling control of flexible structures [2,21,22]. 


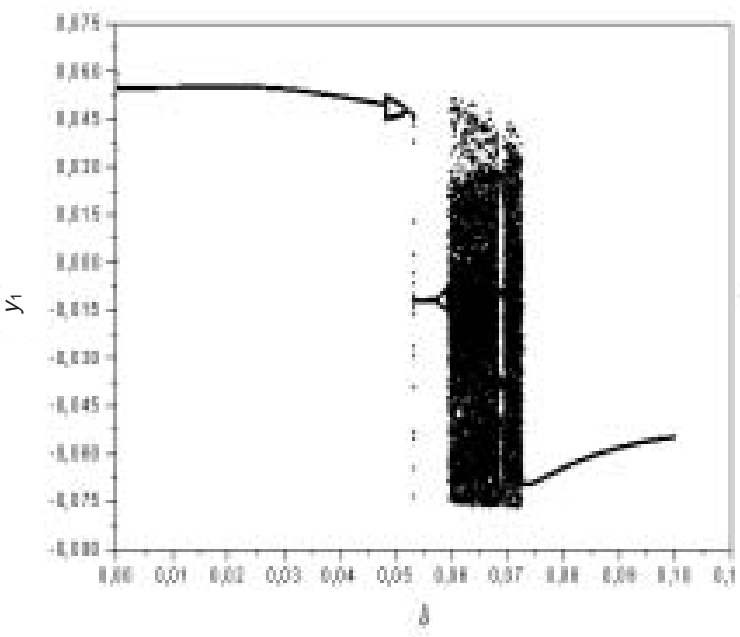

(a)

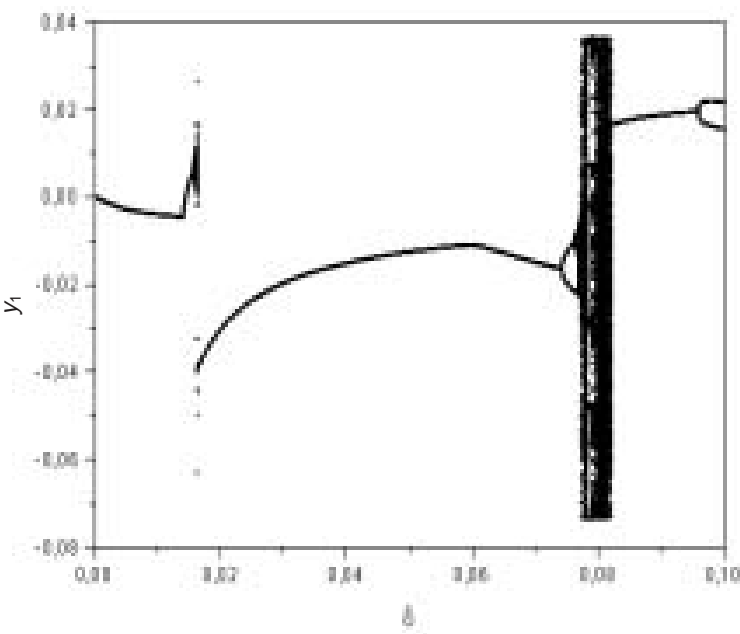

(b)

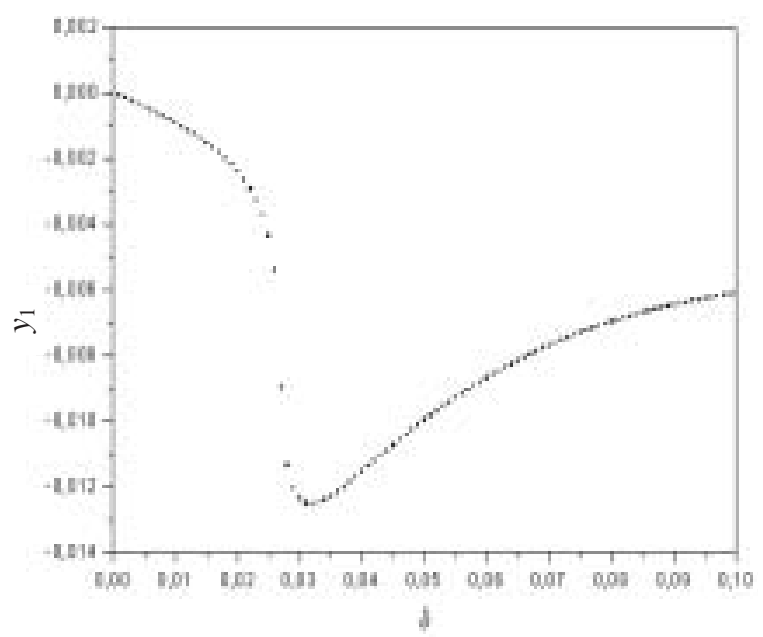

(c)

Fig. 2. Bifurcation diagrams varying parameter $\delta$. (a) $\theta=0.7$; (b) $\theta=1.5$; (c) $\theta=3.5$.

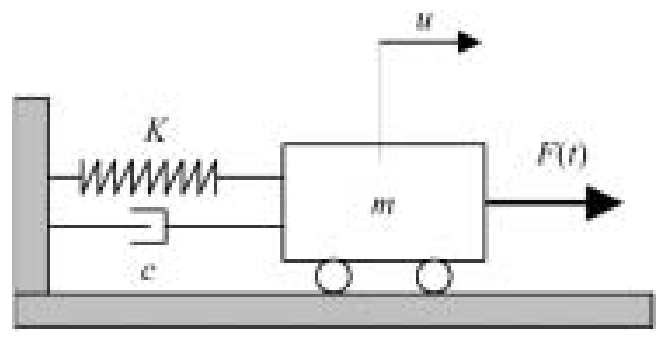

Fig. 1. Shape Memory Oscillator.

The dynamical response of systems with shape memory actuators presents intrinsically nonlinear characteristics and a rich behavior. The dynamical response of shape memory systems is addressed in different references [4,9,24,25,31]. Savi and Braga [26,27] had discussed the chaotic behavior in shape memory oscillators where the restitution force is provided by shape memory helical springs. Savi and Pacheco [28] had studied some characteristic of shape memory oscillators with one and two-degree of freedom, showing the existence of chaos and hyperchaos in these systems. Machado et al. [17] revisited the analysis of coupled shape memory oscillators, considering twodegree of freedom oscillators. Savi et al. [29] analyze a shape memory two-bar truss, showing a very rich response. Recently, some experimental analysis confirms the chaotic behavior of shape memory systems [18]. 


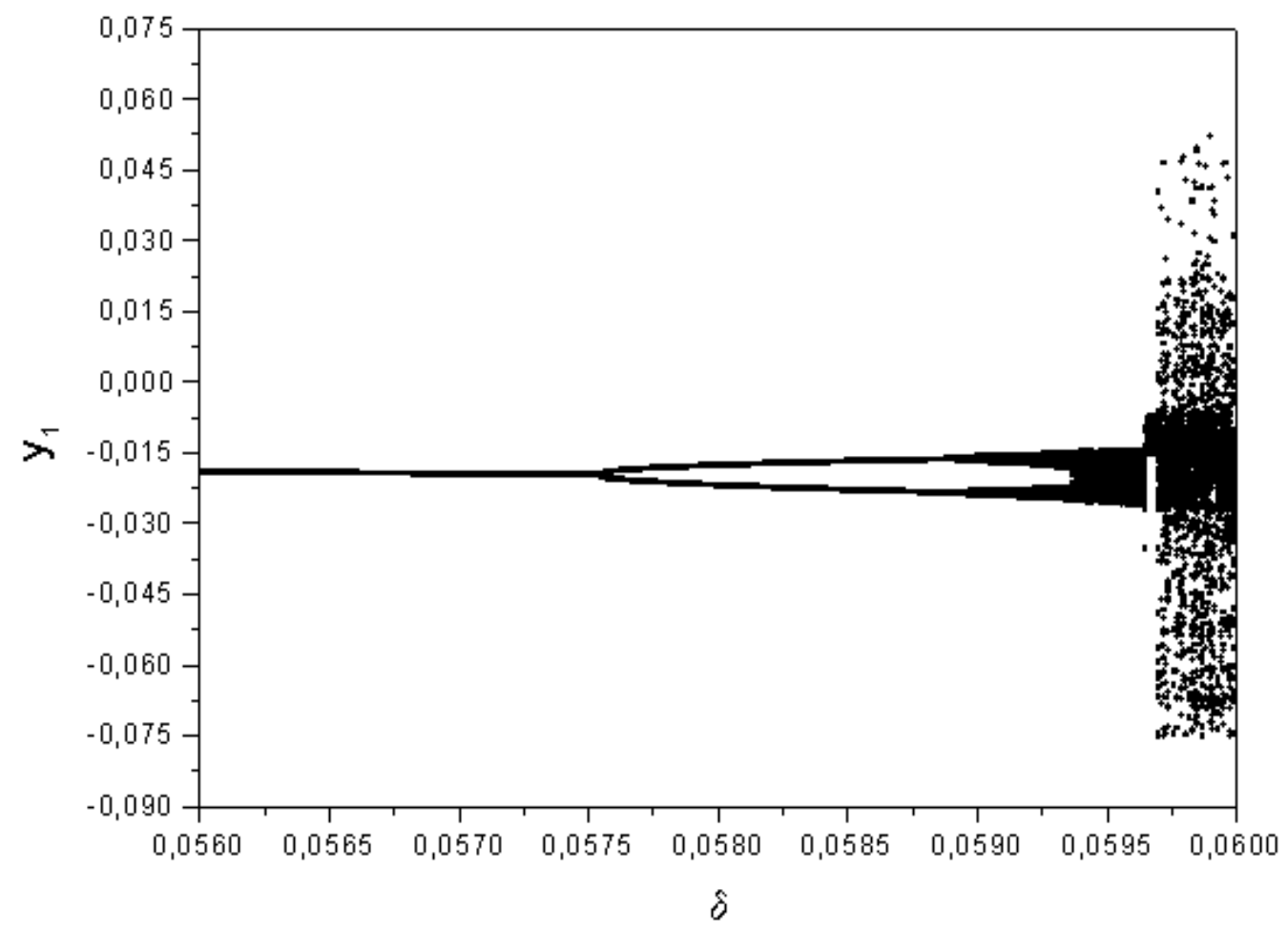

Fig. 3. Bifurcation diagram varying parameter $\delta$ with $\theta=0.7: 0.056 \leqslant \delta \leqslant 0.06$.

This contribution concerns with the chaotic response of shape memory systems analyzing some aspects related to bifurcation phenomenon. The term bifurcation was originally used by Poincaré to describe changes of equilibrium solutions. Nowadays, this term is associated with qualitative changes in the structure of solutions as a consequence of parameter variations, being related to the existence of chaos. This article performs a numerical investigation of a single-degree of freedom shape memory oscillator where the restitution force is described by polynomial constitutive model. Results show period doubling cascades, direct and reverse, and crises on the behavior of the system.

\section{Polynomial model}

The thermomechanical behavior of shape memory alloys may be modeled either by microscopic or macroscopic point of view. Constitutive models consider phenomenological aspects of this behavior and, despite the large number of applications, the modeling of SMA is not well-established [30]. Among all phenomeno- logical theories that describe shape memory effect and pseudoelasticity, polynomial model is the simplest. This model is based on Devonshire theory [7] and the Helmholtz free energy is a function of temperature, $T$, and one-dimensional strain, $E$, i.e., $\Psi=\Psi(E, T)$ [26].

The form of the free energy is chosen in such a way that the minima and maxima points represent stability and instability of each phase of the SMA, respectively. As it is usual on one-dimensional models proposed for SMAs [26], three macroscopic phases are considered: austenite (A) and two variants of martensite $(M+, M-)$. Hence, the free energy is chosen such that for high temperatures, it has only one minimum at vanishing strain, representing the equilibrium of the austenitic phase, $A$. At low temperatures, martensite is stable, and the free energy must have two minima at non-vanishing strains. At intermediate temperatures, the free energy must have equilibrium points corresponding to all phases. These restrictions are satisfied by the following polynomial expression [26]:

$$
\begin{aligned}
\rho \Psi(E, T)= & \frac{1}{2} a\left(T-T_{M}\right) E^{2}-\frac{1}{4} b E^{4} \\
& +\frac{1}{6} e E^{6}
\end{aligned}
$$




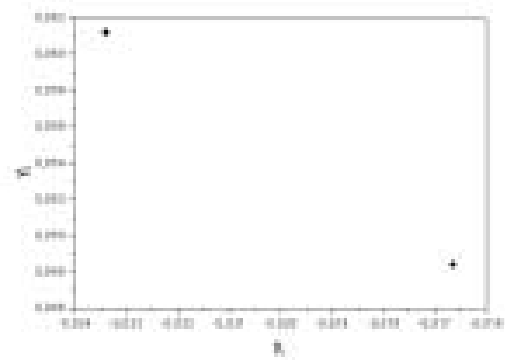

(a)

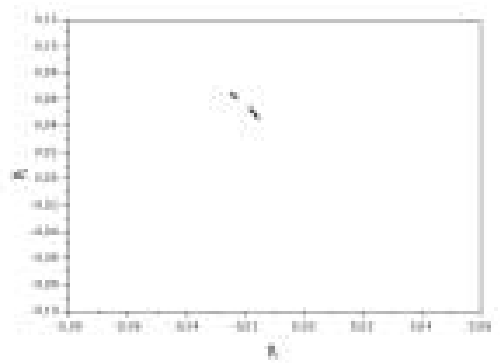

(d)

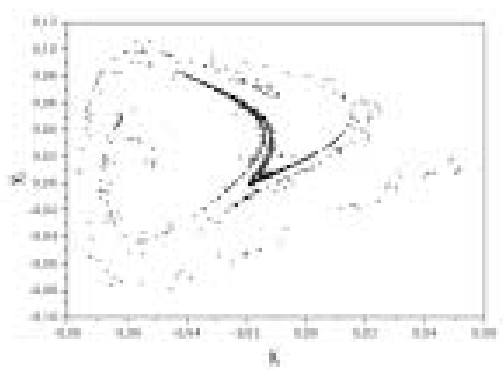

(g)

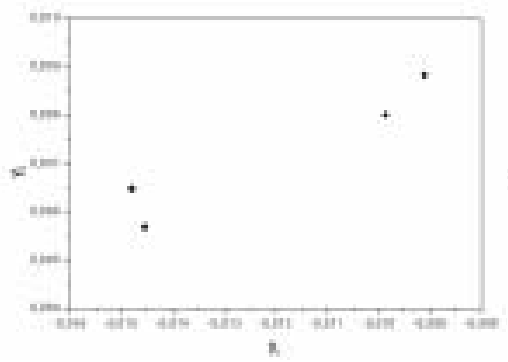

(b)

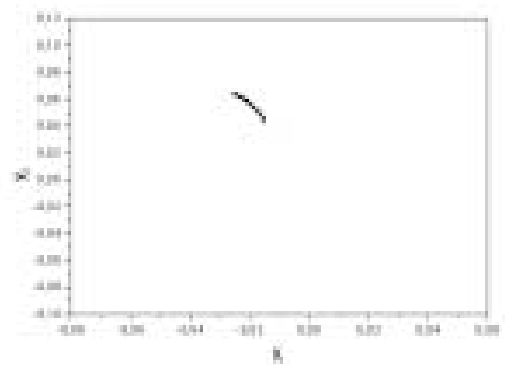

(e)

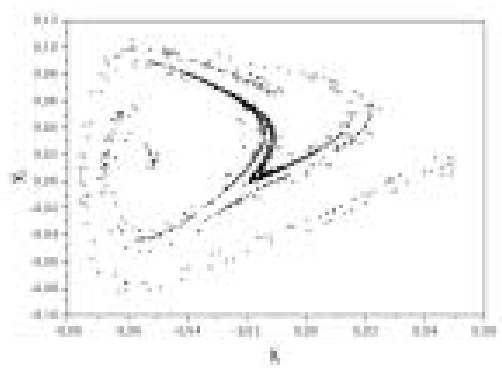

(h)

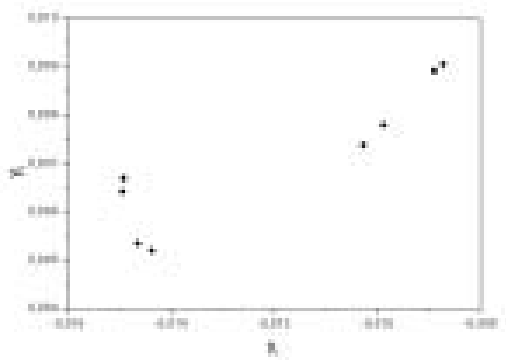

(c)

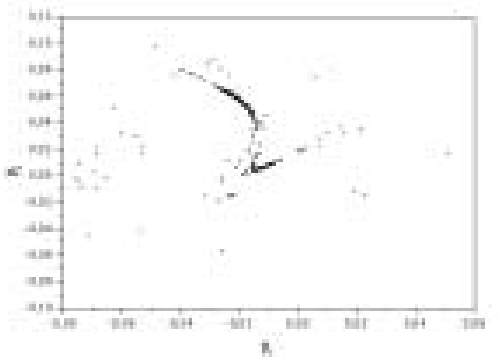

(f)

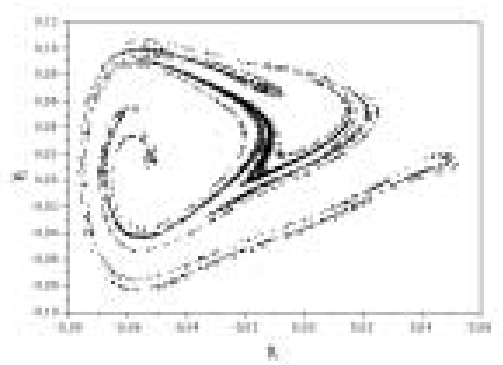

(i)

Fig. 4. Evolution of Poincaré maps due to variation of the force amplitude parameter $\delta$ (birth and formation of the chaotic attractor). (a) $\delta=0.05875$, period-2; (b) $\delta=0.059$, period-4; (c) $\delta=0.0592$, period-8; (d) $\delta=0.0593$, chaos; (e) $\delta=0.0595$, chaos; (f) $\delta=0.05965$, chaos; (g) $\delta=0.059675$, chaos; (h) $\delta=0.05969$, chaos; (i) $\delta=0.06$, chaos.

where $a, b$ and $e$ are positive constants, while $T_{M}$ is the temperature below which the martensitic phase is stable and $\rho$ is the density. If $T_{A}$ is defined as the temperature above which the austenite is stable, and the free energy has only one minimum at zero strains, it is possible to write the following condition,

$$
T_{A}=T_{M}+\frac{1}{4} \frac{b^{2}}{a e}
$$

Therefore, the constant $e$ may be expressed in terms of other constants of the material. By definition [26], the stress-strain relation is obtained from the Helmholtz free energy as follows,

$$
\sigma=\rho \frac{\partial \Psi}{\partial E}=a\left(T-T_{M}\right) E-b E^{3}+e E^{5}
$$

Despite the deceiving simplicity of this model, the authors believe that this analysis is useful to the understanding of the nonlinear dynamics of shape memory systems.

\section{Shape memory oscillator}

Consider a single-degree of freedom oscillator, which consists of a mass $m$ attached to a shape memory element of length $L$ and cross-section area $A$. A linear viscous damper, associated with a parameter $c$, is also considered (Fig. 1). The system is harmonically excited by a force $F=F_{0} \sin (\Omega t)$. 


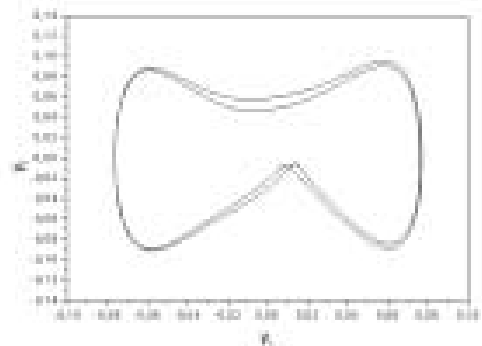

(a)

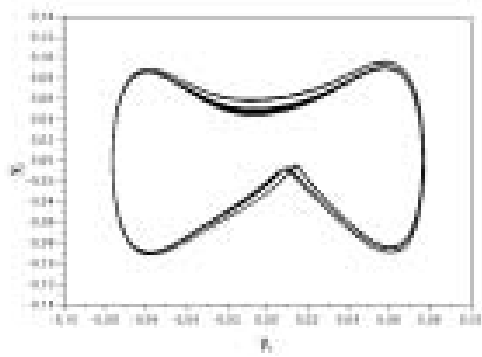

(d)

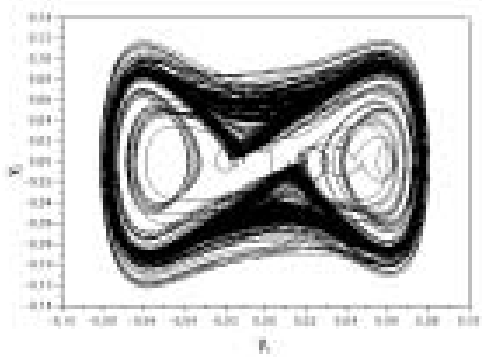

(g)

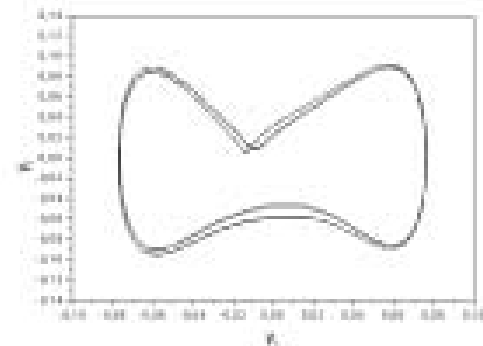

(b)

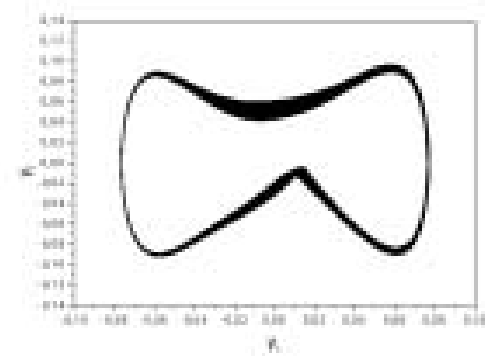

(e)

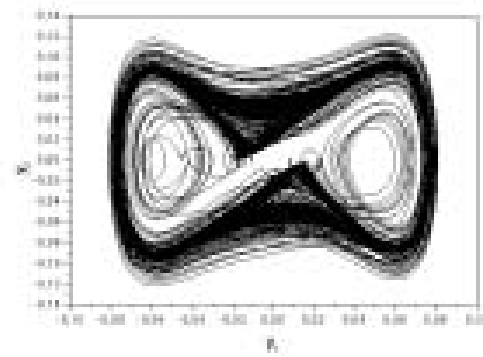

(h)

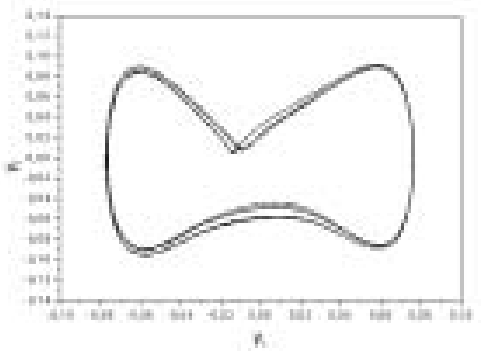

(c)

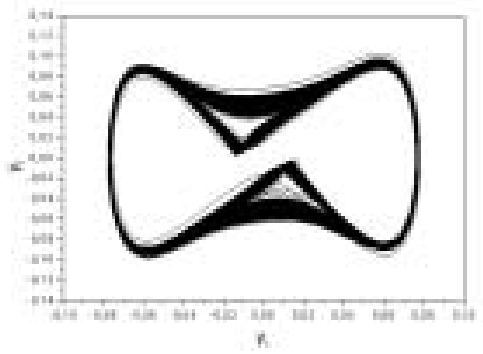

(f)

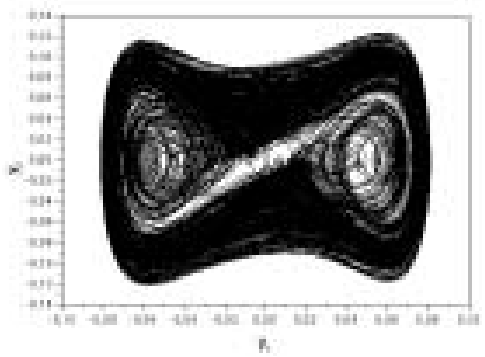

(i)

Fig. 5. Evolution of phase spaces due to variation of the force amplitude parameter $\delta$. (a) $\delta=0.05875$, period-2; (b) $\delta=0.059$, period-4; (c) $\delta=0.0592$, period-8; (d) $\delta=0.0593$, chaos; (e) $\delta=0.0595$, chaos; (f) $\delta=0.05965$, chaos; (g) $\delta=0.059675$, chaos; (h) $\delta=0.05969$, chaos; (i) $\delta=0.06$, chaos.

With these assumptions, equation of motion may be formulated by considering the balance of linear momentum, assuming that the restitution force is provided by a SMA element described by the constitutive equation presented in Eq. (3). Therefore, the following equation of motion is obtained,

$$
\begin{aligned}
& m \ddot{u}+c \dot{u}+\bar{a}\left(T-T_{M}\right) u-\bar{b} u^{3}+\bar{e} u^{5} \\
& =F_{0} \sin (\omega t)
\end{aligned}
$$

where

$$
\bar{a}=\frac{a A}{L} ; \bar{b}=\frac{b A}{L^{3}} ; \bar{e}=\frac{e A}{L^{5}}
$$

In order to obtain a dimensionless equation of motion, system's parameters are defined as follows,

$$
\begin{aligned}
& \xi=\frac{c}{m \omega_{0}} ; \beta=\frac{\bar{b}}{m L^{3} \omega_{0}^{2}} ; \quad \varepsilon=\frac{\bar{e}}{m L^{5} \omega_{0}^{2}} ; \\
& \delta=\frac{F_{0}}{m L \omega_{0}^{2}} ; \quad \omega_{0}^{2}=\frac{\bar{a} T_{M}}{m} ; \varpi=\frac{\omega}{\omega_{0}} ;
\end{aligned}
$$

These definitions allow one to define the following dimensionless variables, respectively related to mass displacement $(U)$, shape memory element temperature $(\theta)$ and time $(\tau)$.

$$
U=\frac{u}{L} ; \quad \theta=\frac{T}{T_{M}} ; \tau=\omega_{0} t
$$

Therefore, the dimensionless equation of motion has the form:

$$
\begin{aligned}
& U^{\prime \prime}+\xi U^{\prime}+(\theta-1) U+\beta U^{3}+\varepsilon U^{5} \\
& =\delta \sin (\varpi \tau)
\end{aligned}
$$




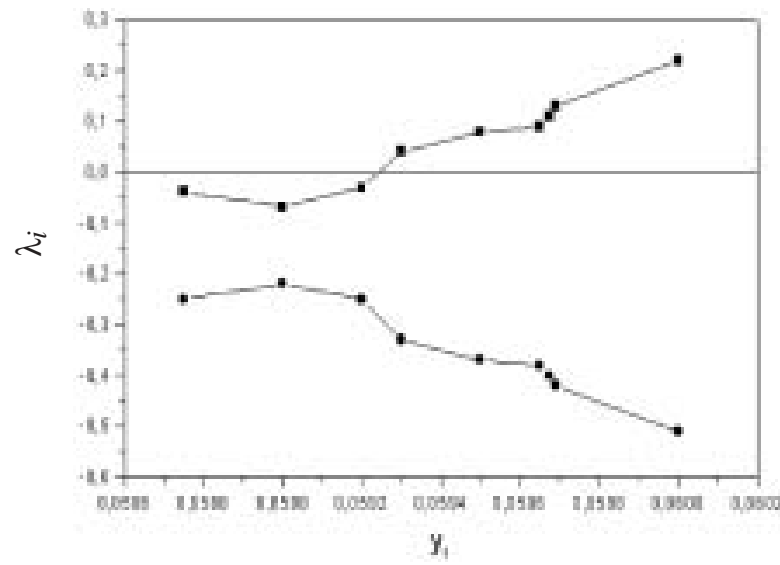

(a)

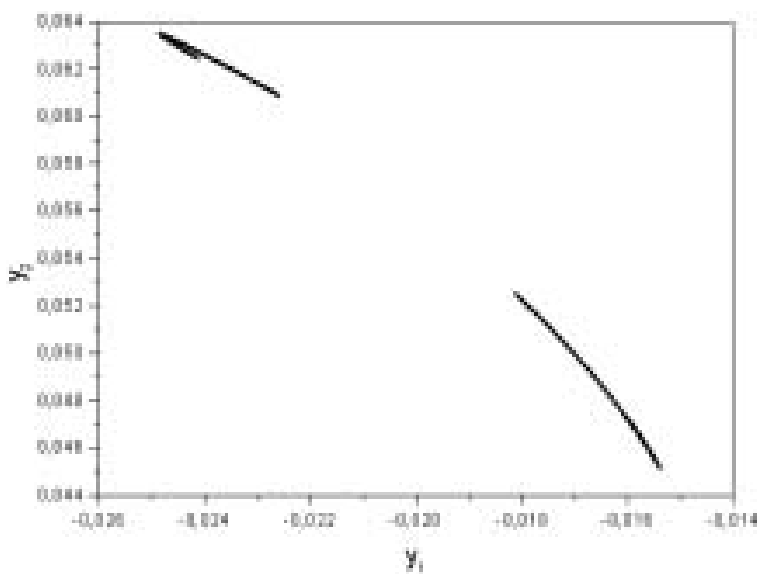

(b)

Fig. 6. (a) Lyapunov exponents for different values of $\delta$ and $\theta=0.7$. (b) Chaotic attractor for $\delta=0.0593$.

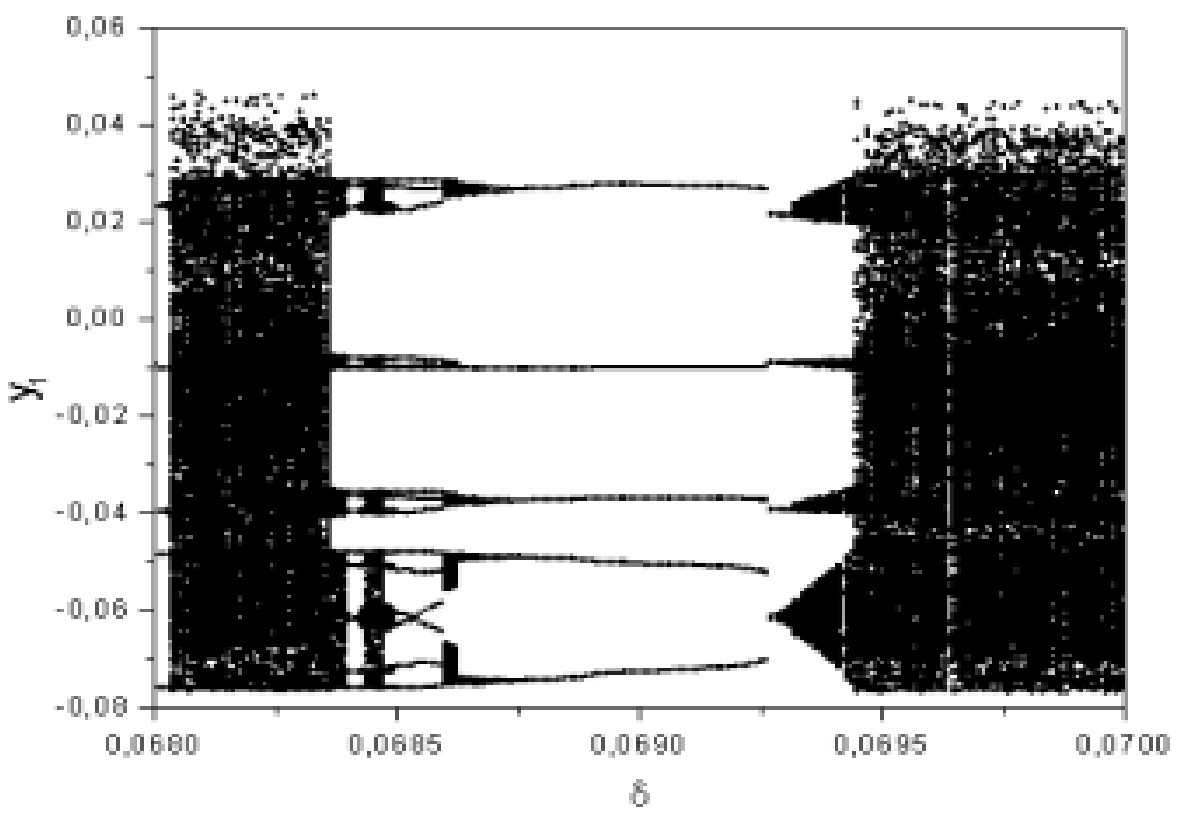

Fig. 7. Periodic window at the interval $0.068 \leqslant \delta \leqslant 0.070$ for $\theta=0.7$.

where derivatives with respect to dimensionless time are represented by,

$$
()^{\prime}=d() / d \tau
$$

This equation of motion can be written in terms of a system of first order differential equations as follows,

$$
\begin{aligned}
& y_{1}^{\prime}=y_{2} \\
& y_{2}^{\prime}=\delta \sin (\varpi \tau)-\xi y_{2}-(\theta-1) y_{1}+\beta y_{1}^{3}-\varepsilon y_{1}^{5}
\end{aligned}
$$

In this article, numerical simulations are performed by the fourth order Runge-Kutta method, with time steps smaller than $\Delta t=2 \pi / 200$. In all studied simulations, it is assumed the parameter values presented in Table 1. Lyapunov exponents characterize chaotic behavior and their estimation is done employing the algorithm due to Wolf et al. [36]. Basically, Lyapunov exponents evaluate the sensitive dependence to initial conditions estimating the exponential divergence of nearby orbits. These exponents have been used as 


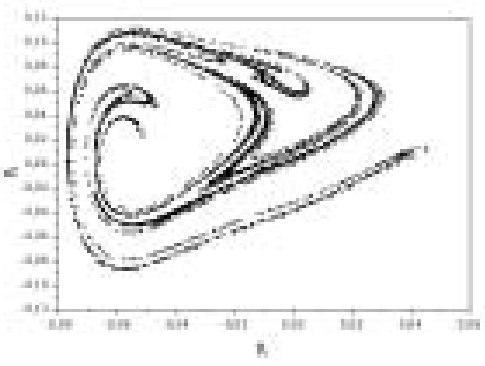

(a)

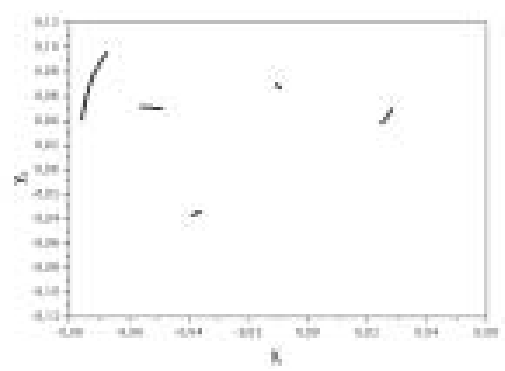

(d)

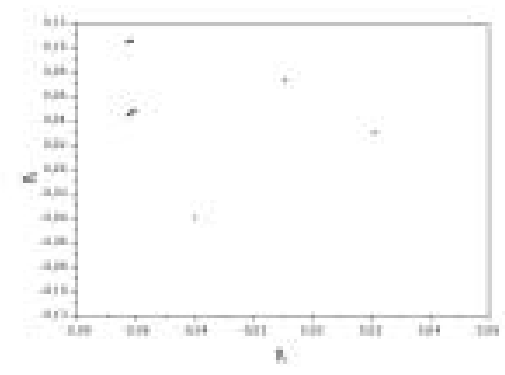

(g)

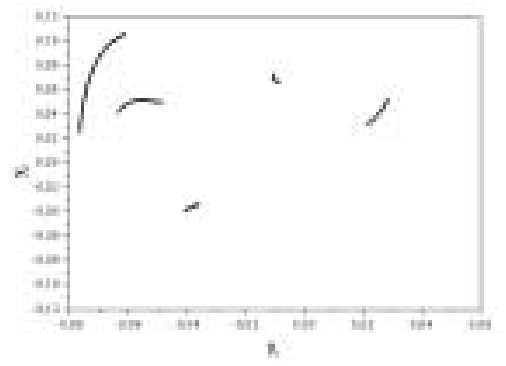

(b)

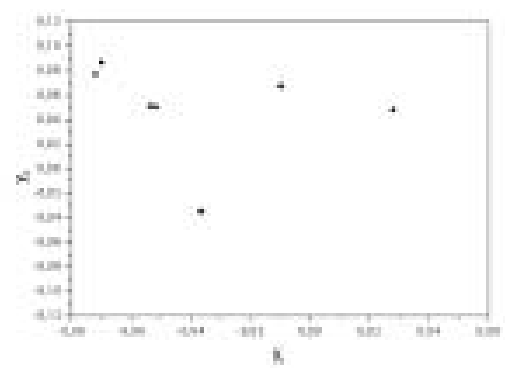

(e)

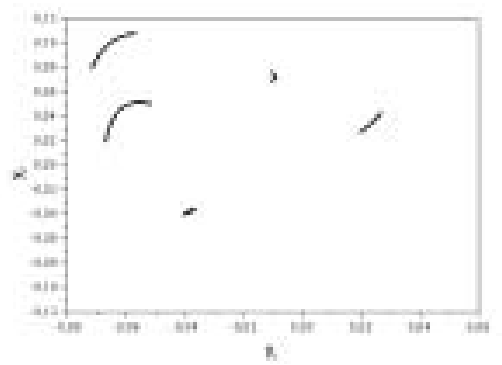

(h)

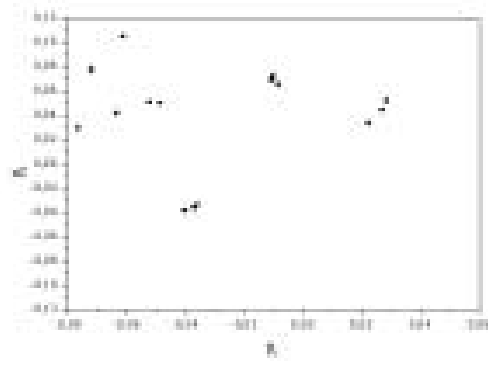

(c)

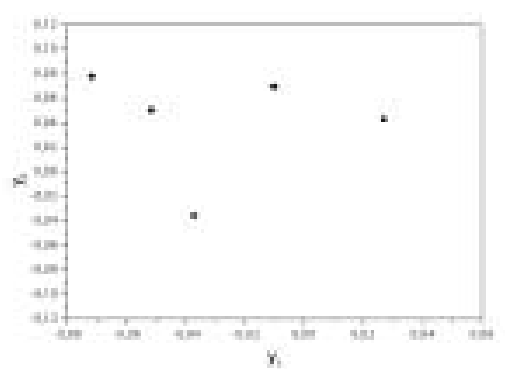

(f)

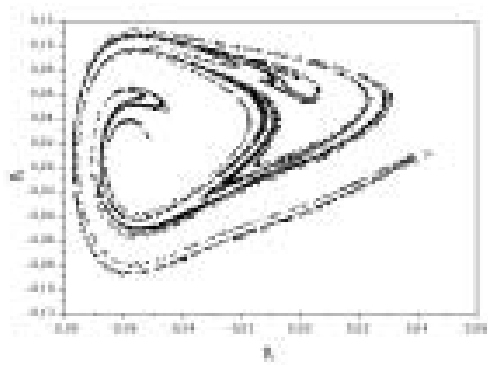

(i)

Fig. 8. Evolution of Poincare maps due to variation of the force amplitude parameter $\delta$ (formation of a periodic window). (a) $\delta=0.0683$, chaos; (b) $\delta=0.06845$, chaos; (c) $\delta=0.0685$, chaos; (d) $\delta=0.0686$, chaos; (e) $\delta=0.0687$, chaos; (f) $\delta=0.0692$, period-5; (g) $\delta=0.0693$, chaos; (h) $\delta=0.0694$, chaos; (i) $\delta=0.0695$, chaos.

Table 1

Shape memory oscillator parameter

\begin{tabular}{cc}
\hline$\xi$ & 0.2 \\
$\varpi$ & 1 \\
$\beta$ & $1.3 \times 10^{3}$ \\
$\varepsilon$ & $4.7 \times 10^{5}$ \\
\hline
\end{tabular}

the most useful dynamical diagnostic tool for chaotic system analysis. The signs of the Lyapunov exponents provide a qualitative picture of the system's dynamics and any system containing at least one positive exponent presents chaotic behavior. The term hyperchaos is used to characterize a behavior where the system has more than one positive exponent.

\section{Bifurcations}

The term bifurcation was originally used by Poincaré to express the change of equilibrium solutions. Nowadays, it is associated with qualitative changes in the structure of solutions, as a consequence of parameter changes, being related to chaos. Bifurcation diagram is a picture that allows a global comprehension of the system dynamics, evaluating the influence of parameter variation in the system behavior. Bifurcation diagram presents a stroboscopic distribution in the response of the system due to a very slow variation of a parameter [32].

This contribution analyzes the structure of bifurcations in a shape memory oscillator considering the 


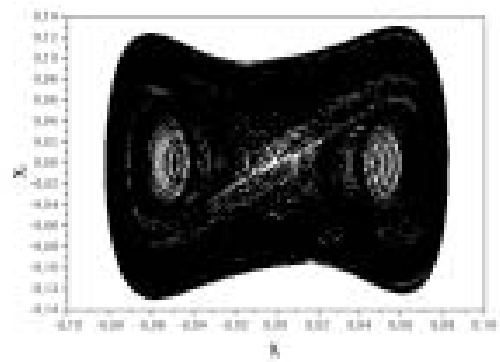

(a)

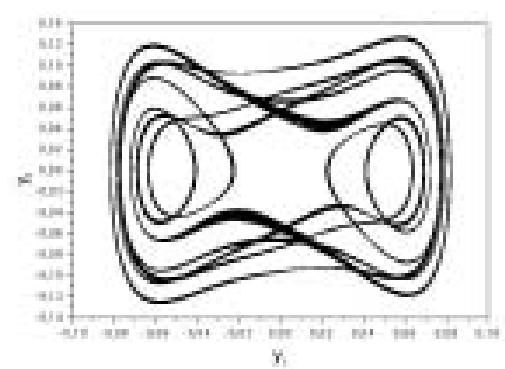

(d)

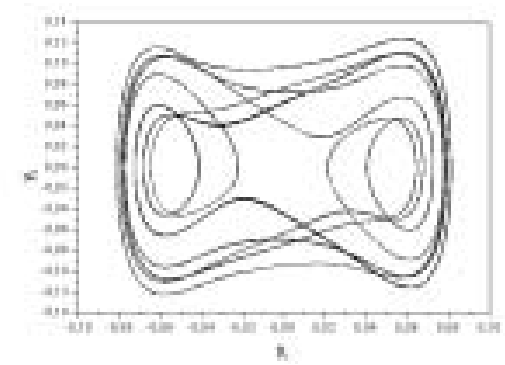

(g)

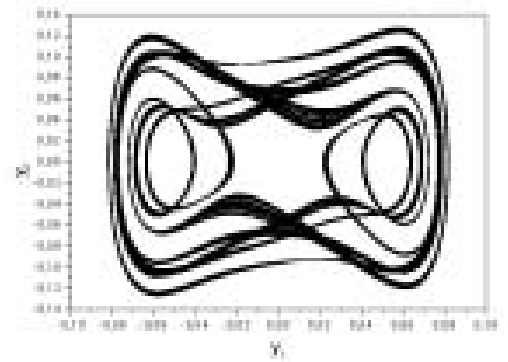

(b)

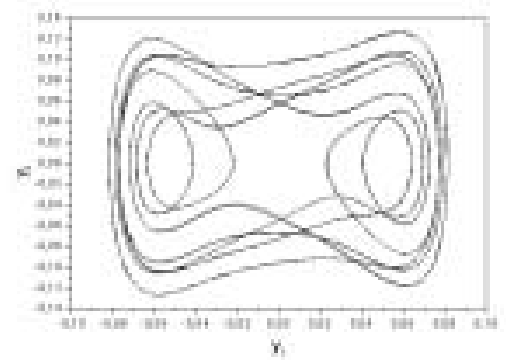

(e)

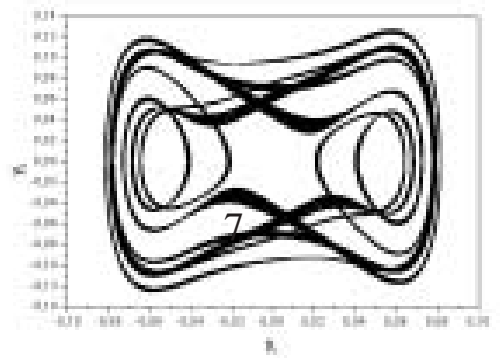

(h)

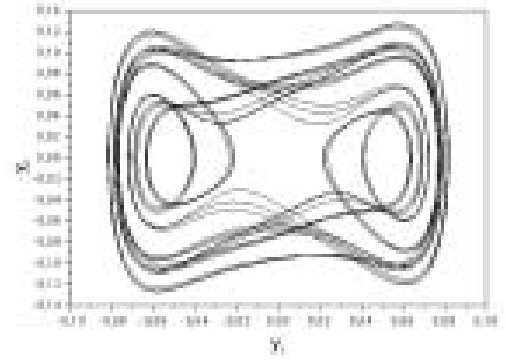

(c)

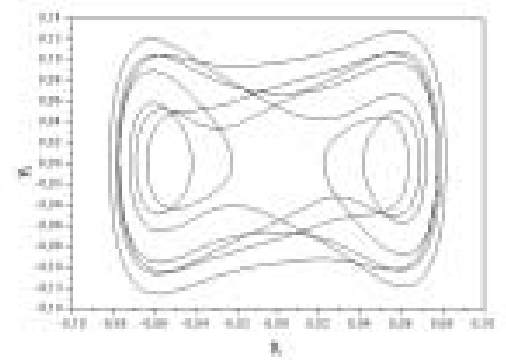

(f)

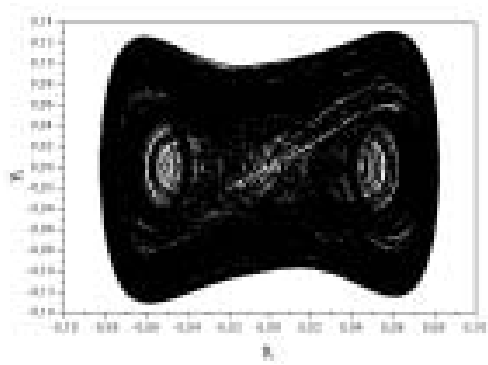

(i)

Fig. 9. Evolution of phase spaces due to variation of the force amplitude parameter $\delta$ (formation of a periodic window). (a) $\delta=0.0683$, chaos; (b) $\delta=0.06845$, chaos; (c) $\delta=0.0685$, chaos; (d) $\delta=0.0686$, chaos; (e) $\delta=0.0687$, chaos; (f) $\delta=0.0692$, period-5; (g) $\delta=0.0693$, chaos; (h) $\delta=0.0694$, chaos, (i) $\delta=0.0695$, chaos.

variation of force amplitude parameter, $\delta$, as well as the parameter related to the temperature of the shape memory element, $\theta$. The analyses of these bifurcations allows one to identify interesting behaviors, such as period doubling cascades, direct and reverse, crises and subductions. The construction of bifurcation diagrams here presented considers the data sampled using Poincaré map, discarding the first 300 periods [20].

\subsection{Bifurcations associated with the force amplitude parameter, $\delta$}

In this section, the influence of variations of the parameter related to the force amplitude, $\delta$, in the system response is discussed. Bifurcation diagrams are evaluated in three distinct temperatures. In the first one, $\theta=0.7$, only the martensitic phase is stable (Fig. 2a). In this situation, regions of bifurcations as well as clouds of points, related to chaos, are observed. In the second situation, an intermediate temperature $(\theta=1.5)$, where both austenite and martensite are stable, is considered (Fig. 2b). In this situation, system response is similar to the previous one. At high temperature $(\theta=3.5)$, Fig. $2 \mathrm{c}$, where only the austenitic phase is stable, changes in parameter value is not related to bifurcation.

The low temperature $(\theta=0.7)$, where martensitic phase is stable, is now in focus. By considering the bifurcation diagram at the interval, $0 \leqslant \delta \leqslant 0.1$, it is possible to observe regions of cloud of points, associ- 


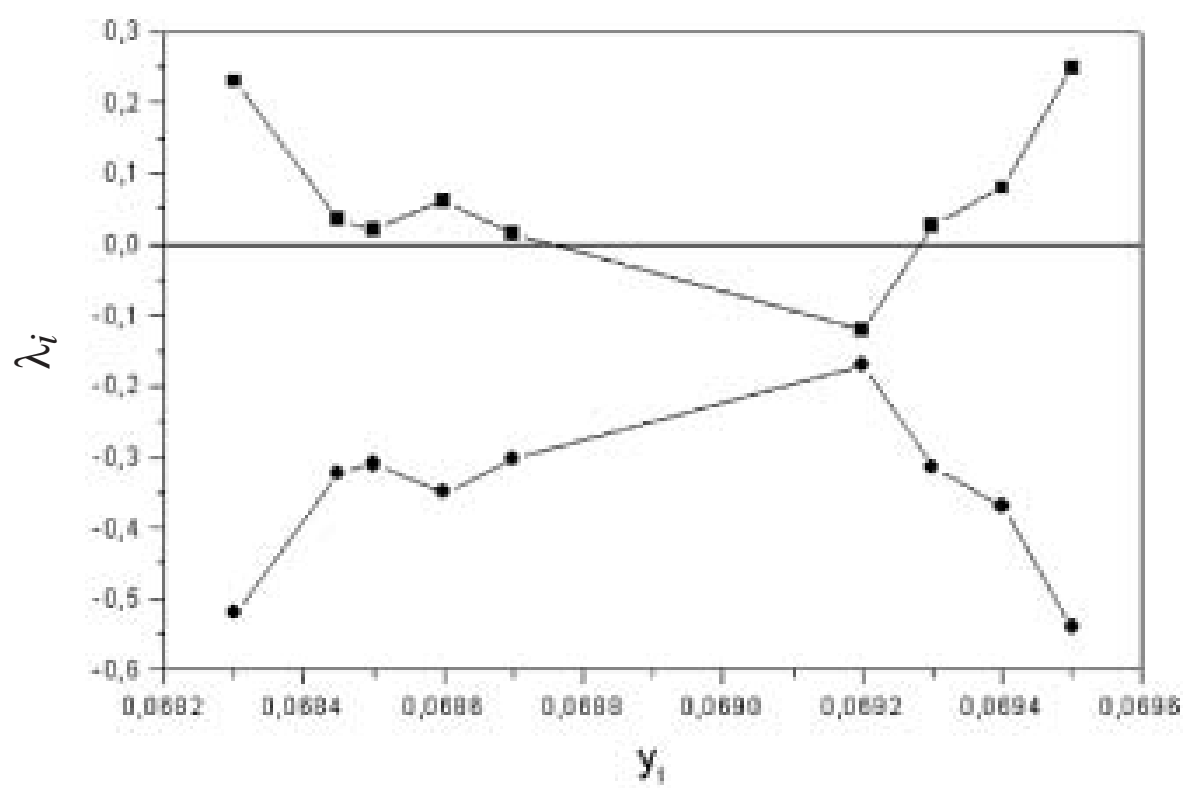

Fig. 10. Lyapunov exponents for different values of $\delta$ and $\theta=0.7$.

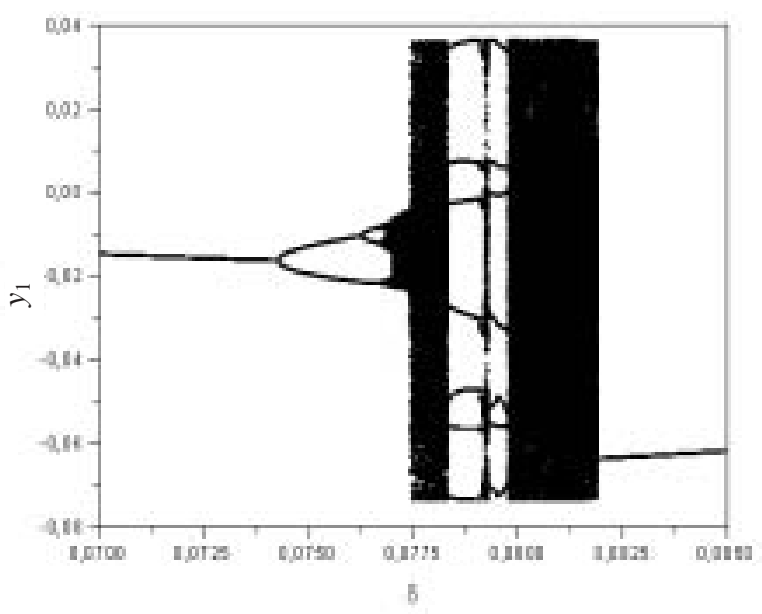

(a)

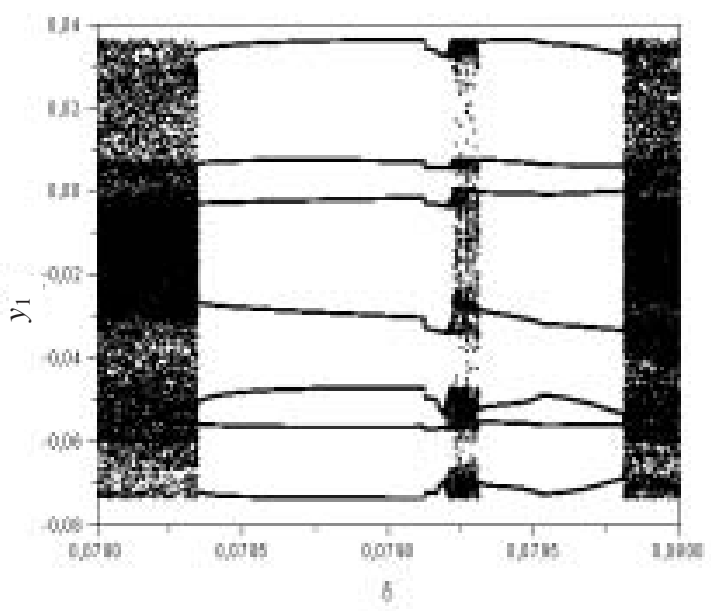

(b)

Fig. 11. Bifurcation diagram varying force amplitude parameter $\delta$ with $\theta=1.5$ : (a) $0.07 \leqslant \delta \leqslant 0.0850$. (b) $0.078 \leqslant \delta \leqslant 0.08$.

ated with chaotic behavior. Periodic windows separate these chaotic regions (Fig. 2a). When $\delta=0.0575$, the system loses stability and there is a period doubling bifurcation. When $\delta=0.05875$, there is another bifurcation. These bifurcations repeat, over and over, making a period doubling cascade, which is an infinite sequence of period doubling bifurcations up to chaos [1]. Figure 3 presents a zoom at the interval $0.056 \leqslant \delta \leqslant 0.06$. This enlargement allows one to observe the transition from order to chaos. When $\delta=0.0593$, there is a sudden birth of a chaotic attractor, associated with the existence of a positive Lyapunov exponent. This phenomenon is known as crisis. Grebogi et al. [11] defined crisis as a collision between a chaotic attractor with an unstable orbit or a fixed point. The crisis that happened in the shape memory oscillator when $\delta=0.0593$ is known as boundary crisis, which is related with the birth of the chaotic attractor and its basin of attraction.

Figure 4 shows a sequence of Poincaré maps associated with different values of the parameter $\delta$. Fig- 

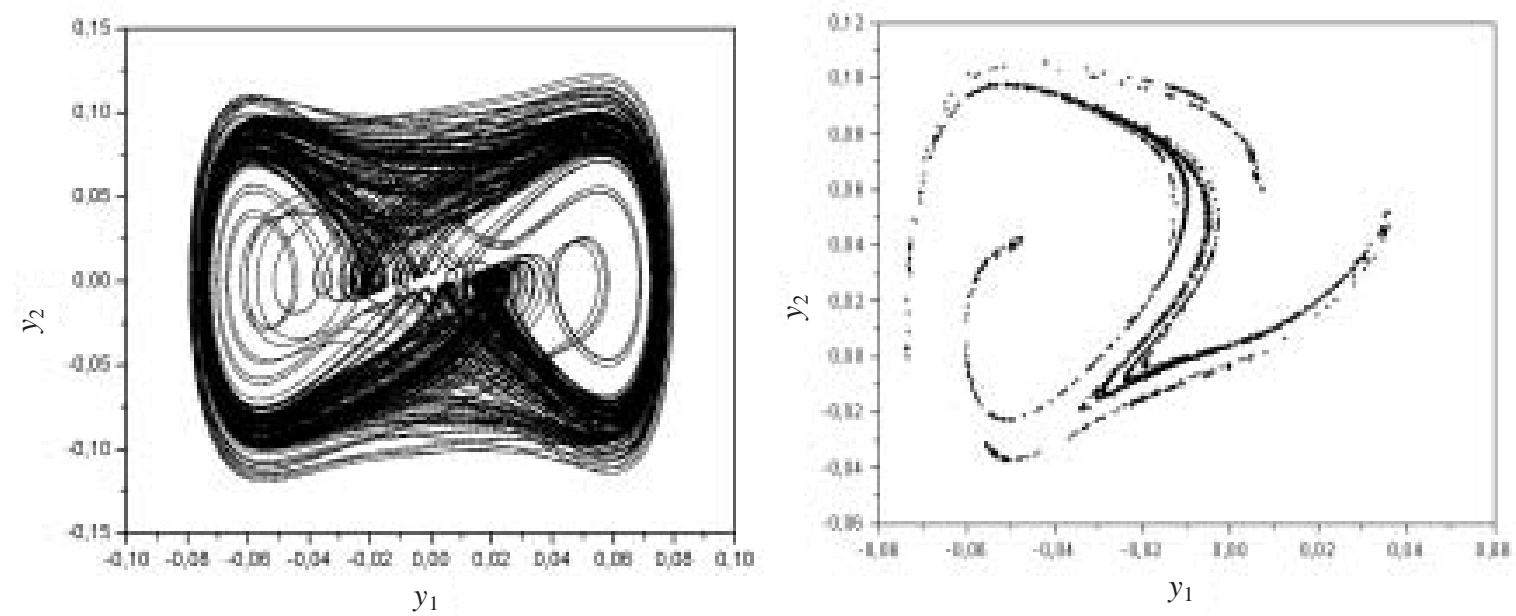

Fig. 12. Response for $\delta=0.0777$ and $\theta=1.5$.

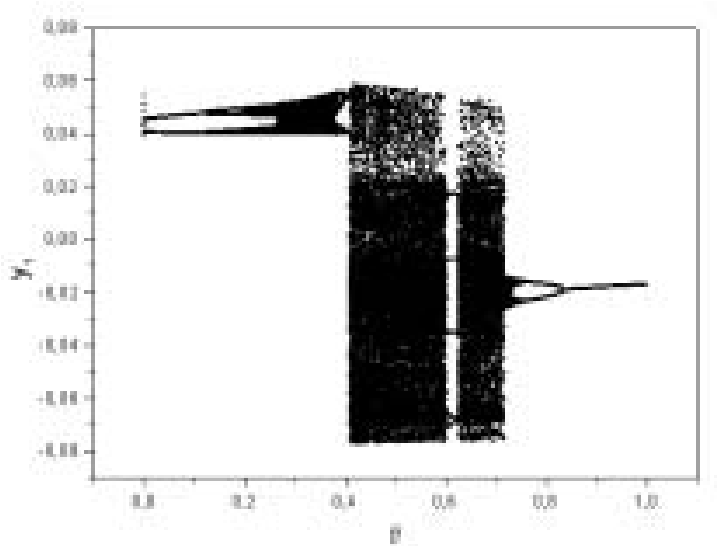

(a)

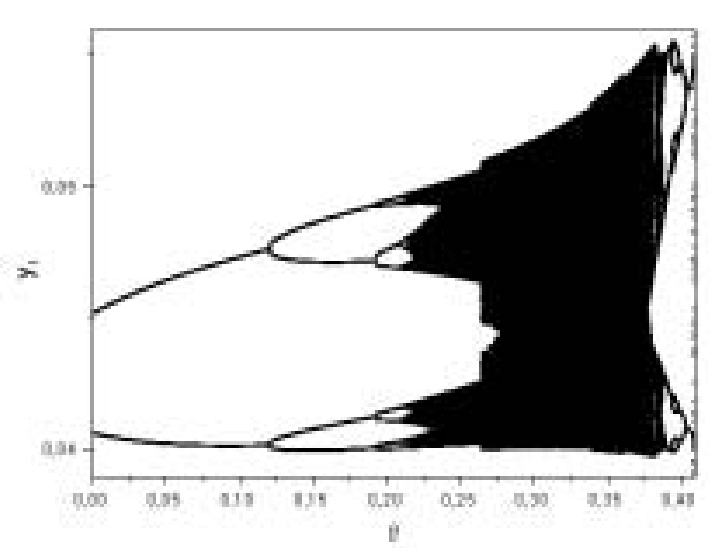

(b)

Fig. 13. Bifurcation diagram varying temperature parameter $\theta$. (a) $0<\theta \leqslant 1$; (b) $0<\theta \leqslant 0.405$.

ures $4 \mathrm{a}, 4 \mathrm{~b}, 4 \mathrm{c}$ are related to periodic behavior of period 2, 4 and 8, respectively. From Fig. 4 d to $4 \mathrm{i}$, when $\delta \geqslant 0.0593$, there are chaotic behaviors characterized by one positive Lyapunov exponent. Figure 4d shows the birth of the chaotic attractor, while Figs 4e, 4f, 4g, $4 \mathrm{~h}$ and $4 \mathrm{i}$ present its formation. This process of attractor formation is understood as interior crisis of the system, which is related to sudden changes in the length or form of the attractor. Figure 5 shows phase spaces related to the behaviors presented in Fig. 4.

Lyapunov exponents for different values of parameter $\delta$ are analyzed in Fig. 6a, making noticeable the moment when the motion become chaotic. A zoom of the strange attractor of Fig. $4 d$, in the instant when chaos appears, is shown in Fig. 6b.
By considering a new enlargement of bifurcation diagram at the interval $0.068 \leqslant \delta \leqslant 0.07$, it is possible to observe a periodic window (Fig. 7). The formation of this periodic window is associated with a phenomenon known as subduction. Grebogi et al. [11] define subduction as the birth of a non-chaotic attractor within a chaotic attractor, where the first take the place of the last one. The difference between boundary crisis and subduction is characterized by the fact that the last one does not destroy the basin of attraction. In this periodic window, in particular, one can note the presence of a period- 5 behavior at its central region, in opposite to chaos in its edges. An evolution of Poincaré maps due to different values of $\delta$ in the periodic window is presented in Fig. 8. Figure 9 presents phase spaces for 


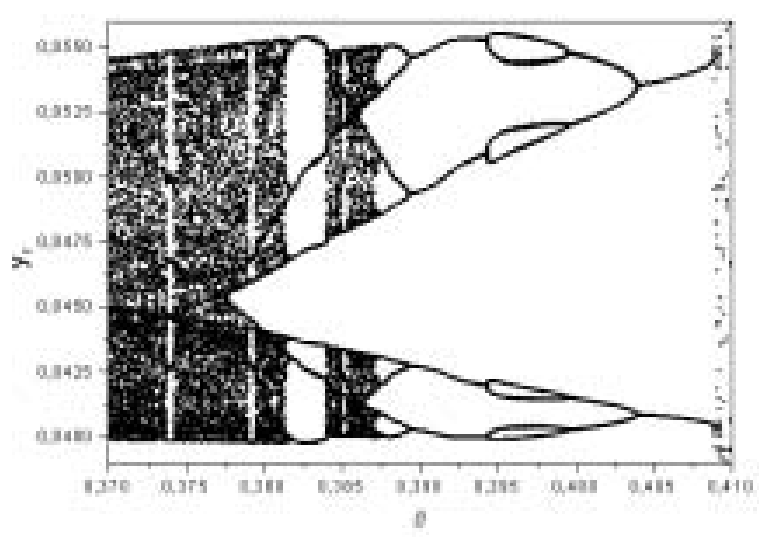

(a)

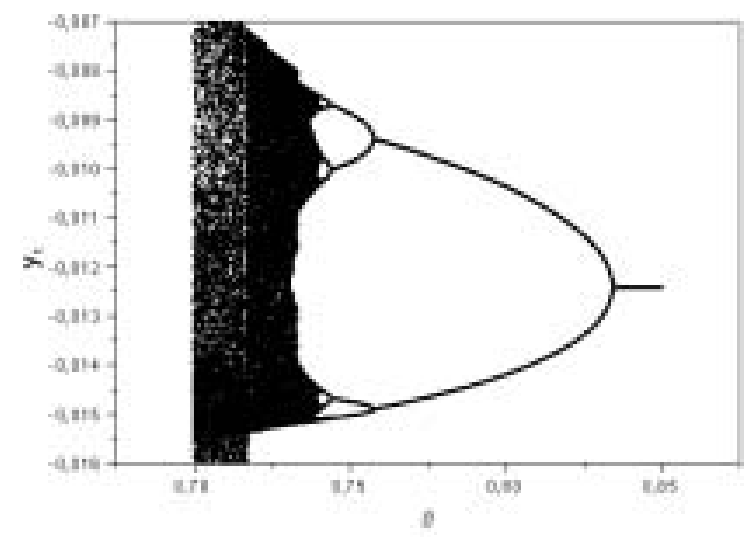

(b)

Fig. 14. Bifurcation diagram varying temperature parameter $\theta$. (a) $0.37 \leqslant \theta \leqslant 0.41$; (b) $0.70 \leqslant \theta \leqslant 0.85$.

the same values. The evolution of Lyapunov exponents is shown in Fig. 10.

The intermediate temperature $(\theta=1.5)$ is now treated. At this temperature, both austenite and martensite are stable. Figure 11 presents enlargements of the bifurcation diagram shown in Fig. 2b. Bifurcation and crisis phenomena are similar to the one at $\theta=0.7$. Figure 12 shows the response of this system for $\delta=0.0777$.

\subsection{Bifurcations related to the temperature parameter $\theta$}

The analysis of bifurcations associated with variations of the temperature parameter $\theta$ is now in focus. This analysis is an important tool to study the dynamical behavior of SMA engineering applications since temperature is the essential control parameter for SMA applications. It should be pointed out that the temperature variation is associated with phase transformations that modify the system characteristics. Figure 13a presents the bifurcation diagram due to the variation of $\theta$ in the interval $0<\theta \leqslant 1$, with $\delta=0.06$. Different kinds of responses can be identified and, when $\theta>1$ (situations where austenite becomes stable), the system does not present chaotic behavior anymore. Figure 13b shows a zoom of the bifurcation diagram in the interval $0<\theta \leqslant 0.405$. For low temperatures, there are two periodic orbits. These orbits are stable until $\theta=0.12$ is reached. At this moment, the system loses its stability, presenting a period doubling. Afterwards, the four stable orbits lose their stability again, and another period doublings are noted. This sequence is repeated over and over, making up a period doubling cascade.
By enlarging Fig. 13b at the interval $0.37 \leqslant \theta \leqslant 0.41$ (Fig. 14a), it is observed a succession of periodic windows within chaotic regions until $\theta=0.3875$ is reached. Afterwards, when $0.3875 \leqslant \theta \leqslant 0.409$, periodic behaviors appear, where there are period doubling bifurcations, direct and reverse. When $\theta=0.409$, crisis arises in the system and, again, chaos. Considering the interval $0.70 \leqslant \theta \leqslant 0.85$, shown in Fig. $14 \mathrm{~b}$, one can see the moment that chaos is replaced by a reverse period doubling cascade $(\theta=0.717)$. This reverse cascade continues to present bifurcations until a period-1 response is reached. From this moment on, the system presents only periodic behaviors. It is worthwhile to mention that the arising of this reverse cascade is related to a crisis as well.

The evolution of the attractors related to this crisis is similar to the one in the case of bifurcations due to the variation of force amplitude parameter $\delta$. Figure 15 presents the evolution of Poincaré maps considering different values of parameter $\theta$, at the interval $0.37 \leqslant$ $\theta \leqslant 0.41$ (Fig. 14a). On the other hand, Fig. 16 presents phase spaces related to the same values of $\theta$.

\section{Conclusions}

This contribution analyses some aspects associated with bifurcation phenomenon in shape memory systems. A single-degree of freedom mechanical oscillator where the restitution force is described by a polynomial constitutive model is considered. Results of numerical simulations indicate that this system has a very rich behavior with different kinds of responses. $\mathrm{Bi}$ furcation analysis is carried out considering the struc- 


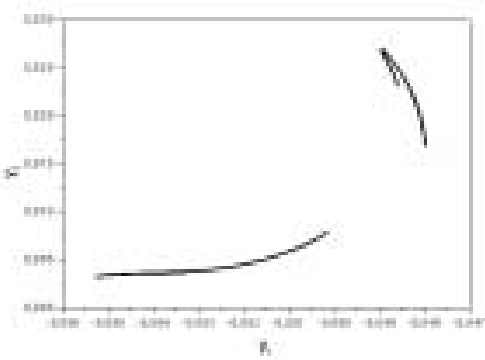

(a)

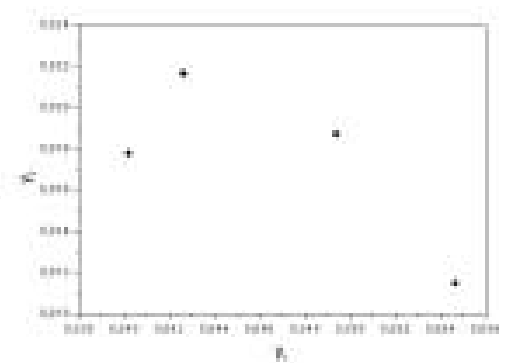

(d)

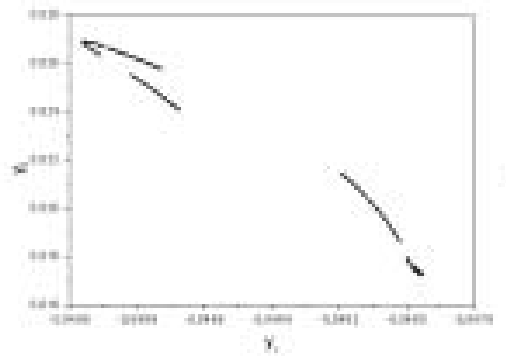

(b)

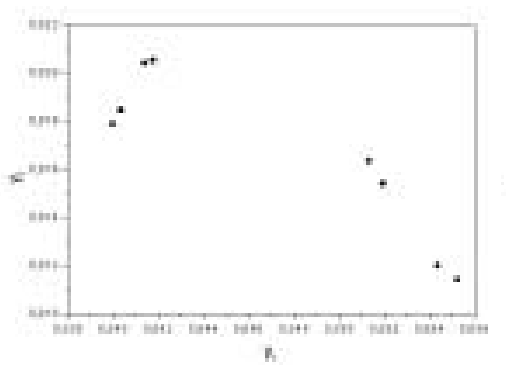

(e)

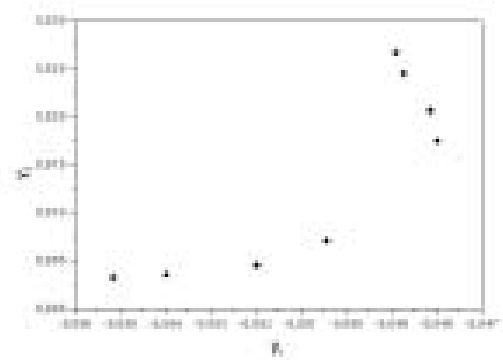

(c)

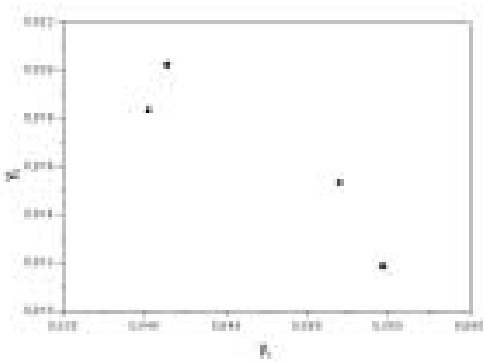

(f)

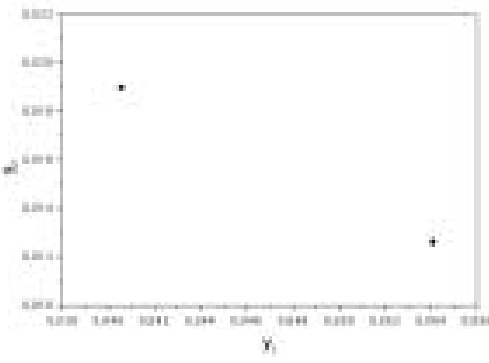

(g)

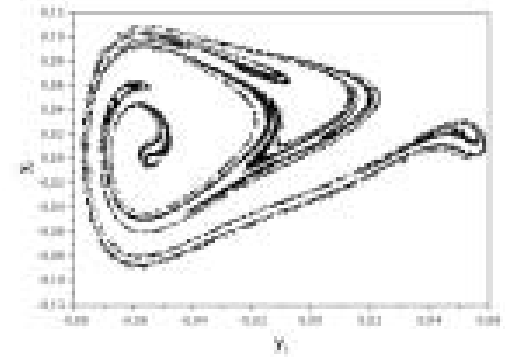

(h)

Fig. 15. Evolution of Poincaré maps due to variation of the temperature parameter $\theta$ (formation of a periodic window). (a) $\theta=0.386$, chaos; (b) $\theta=0.387$, chaos; (c) $\theta=0.3875$, period-8; (d) $\theta=0.389$, period-4; (e) $\theta=0.38975$, period-8; (f) $\theta=0.4$, period-4; (g) $\theta=0.408$, period-2; (h) $\theta=0.409$, chaos.

ture of bifurcations varying force amplitude parameter, $\delta$, as well as the parameter related to the temperature of shape memory element, $\theta$. The analyses of these bifurcations allows one to identify interesting behaviors, such as period doubling cascades, direct and reverse, crises and subductions. Therefore, the response of SMA devices subjected to dynamic loadings can be very complex being of special interest to be investigated. Moreover, the possibility of chaotic response should be considered in the design of these systems.

\section{Acknowledgments}

The authors acknowledge the support of the Brazilian Agencies CNPq and CAPES.

\section{References}

[1] K.T. Alligood, T.D. Sauer and J.A. Yorke, Chaos: An Introduction to Dynamical Systems, Springer-Verlag, 1997.

[2] V. Birman, Review of Mechanics of Shape Memory Alloy Structures, Applied Mechanics Review 50 (1997), 629-645.

[3] T. Borden, Shape Memory Alloys: Forming a Tight Fit, Mechanical Engineering, 1991, 66-72.

[4] M. Collet, E. Foltête and C. Lexcellent, Analysis of the Behavior of a Shape Memory Alloy Beam Under Dynamical Loading, European Journal of Mechanics A - Solids 20 (2001), 615-630.

[5] K.K. Denoyer, R. Scott Erwin and R. Rory Ninneman, Advanced Smart Structures Flight Experiments for Precision Spacecraft, Acta Astronautica 47 (2000), 389-397.

[6] T. Duerig, A. Pelton and D. Stöckel, An overview of Nitinol Medical Applications, Materials Science and Engineering A 273-275 (1999), 149-160. 


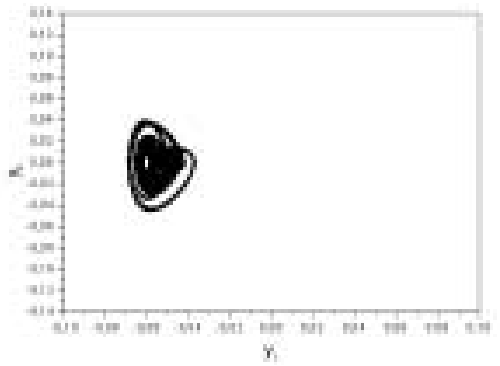

(a)

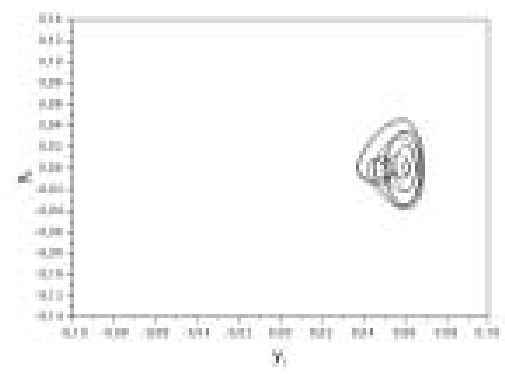

(d)

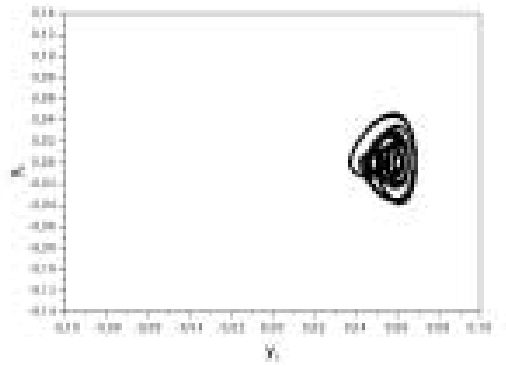

(b)

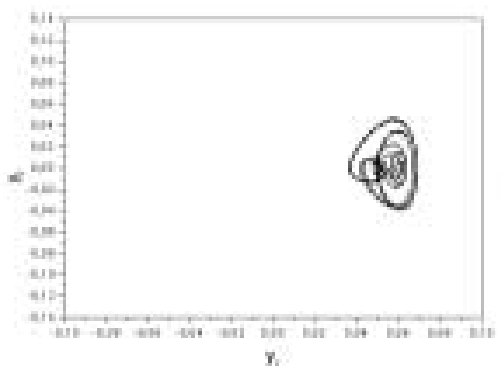

(e)

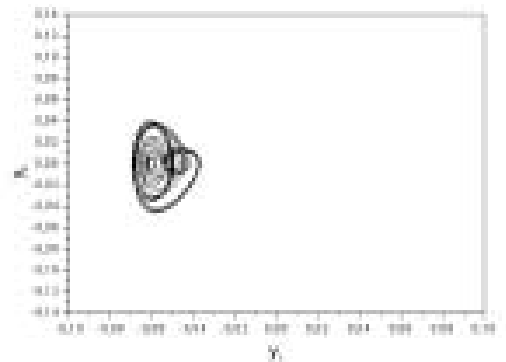

(c)

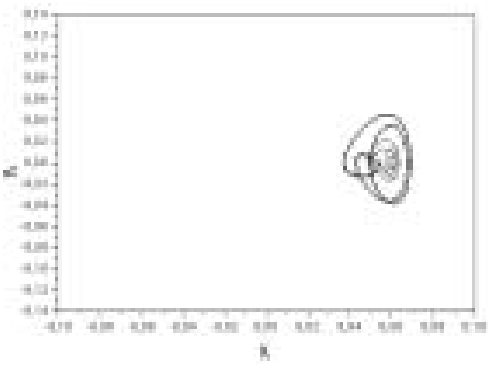

(f)

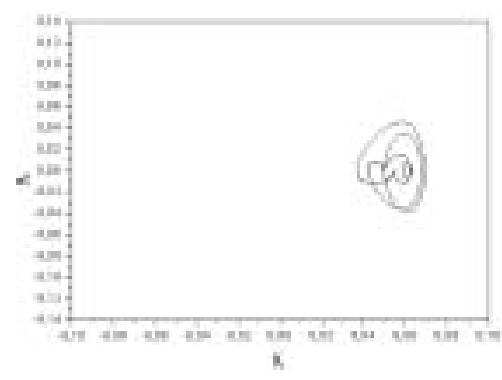

(g)

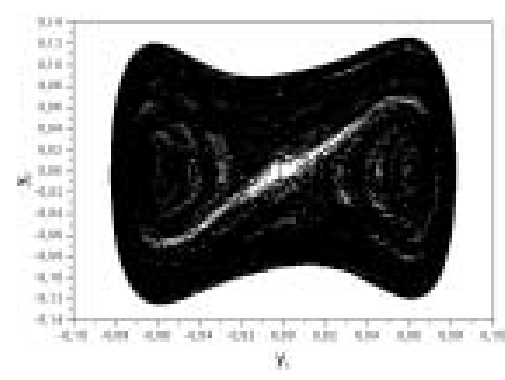

(h)

Fig. 16. Evolution of Poincare maps due to variation of the temperature parameter $\theta$ (formation of a periodic window). (a) $\theta=0.386$, chaos; (b) $\theta=0.387$, chaos; (c) $\theta=0.3875$, period-8; (d) $\theta=0.389$, period-4; (e) $\theta=0.38975$, period-8; (f) $\theta=0.4$, period-4; (g) $\theta=0.408$, period-2; (h) $\theta=0.409$, chaos.

[7] F. Falk, Model Free Energy, Mechanics, and Thermodynamics of Shape Memory Alloys, Acta Metalurica 28 (1980), 17731780 .

[8] H. Fujita and H. Toshiyoshi, Micro Actuators and Their Applications, Microelectronics Journal 29 (1998), 637-640.

[9] F. Gandhi and G. Chapuis, Passive Damping Augmentation of a Vibrating Beam Using Pseudoelastic Shape Memory Alloy, Journal of Sound and Vibration 250(3) (2002), 519-539.

[10] L.J. Garner, L.N. Wilson, D.C. Lagoudas and O.K. Rediniotis, Development of a Shape Memory Alloy Actuated Biomimetic Vehicle, Smart Materials \& Structures 9(5) (2001), 673-683.

[11] C. Grebogi, E. Ott and J.A. Yorke, Crises, Sudden Changes in Chaotic Attractors, and Transient Chaos, Physica D 7 (1983), 181-200.

[12] E. Kibirkstis, R. Liaudinskas, D. Pauliukaitis and K. Vaitasius, Mechanisms with Shape Memory Alloy, Journal de Physique IV C5 (1987), 633-636.
[13] C.A.P.L. La Cava, E.P. Silva, L.G. Machado, P.M.C.L. Pacheco and M.A. Savi, Modeling of a Shape Memory Preload Device for Bolted Joints, Proceedings of National Congress of Mechanical Engineering (CONEM 2000 - ABCM), Natal, Brazil (in portuguese), 2000.

[14] D.C. Lagoudas, O.K. Rediniotis and M.M. Khan, Applications of Shape Memory Alloys to Bioengineering and Biomedical Technology, Proceedings of 4th International Workshop on Mathematical Methods in Scattering Theory and Biomedical Technology, October 1999, Perdika, Greece.

[15] L.G. Machado and M.A. Savi, Odontological Applications of Shape Memory Alloys, Revista Brasileira de Odontologia 59(5) (2002), 302-306 (in portuguese).

[16] L.G. Machado and M.A. Savi, Medical Applications of Shape Memory Alloys, Brazilian Journal of Medical and Biological Research 36(6) (2003), 683-691.

[17] L.G. Machado, M.A. Savi and P.M.C.L. Pacheco, Nonlinear Dynamics and Chaos in Coupled Shape Memory Oscillators, 
International Journal of Solids and Structures 40(19) (2003), 5139-5156.

[18] M.J. Mosley and C. Mavroidis, Experimental Nonlinear Dynamics of a Shape Memory Alloy Wire Bundle Actuator, Journal of Dynamic Systems, Measurement and Control $\mathbf{1 2 3}$ (2001), 103-123.

[19] P.M.C.L. Pacheco and M.A. Savi, A Non-Explosive Release Device for Aerospace Applications using Shape Memory Alloys, Proceedings of XIV the Brazilian Congress of Mechanical Engineering (COBEM 97 - ABCM), Bauru, Brazil, 1997.

[20] T.S. Parker and L.O. Chua, Practical Numerical Algorithm for Chaotic Systems, Springer-Verlag, 1989.

[21] M. Pietrzakowski, Natural Frequency Modification of Thermally Activated Composite Plates, Mec. Ind. 1 (2000), 313320.

[22] C.A. Rogers, Intelligent Materials, Scientific American, September, 1995, 122-127.

[23] C.A. Rogers, C. Liang and C.R. Fuller, Modeling of Shape Memory Alloy Hybrid Composites for Structural Acoustic Control, Journal of Acoustic Society of America (89) (1991), 210-220.

[24] S. Saadat, J. Salichs, M. Noori, Z. Hou, H. Davoodi, I. Bar-On, Y. Suzuki and A. Masuda, An overview of vibration and seismic applications of NiTi shape memory alloy, Smart Materials \& Structures 11(2) (2002), 218-229.

[25] J. Salichs, Z. Hou and M. Noori, Vibration Suppression of Structures Using Passive Shape Memory Alloy Energy Dissipation Devices, Journal of Intelligent Material Systems and Structures 12(10) (2001), 671-680.

[26] M.A. Savi and A.M.B. Braga, Chaotic Vibrations of na Oscillator with Shape Memory, Journal of the Brazilian Society of Mechanical Sciences and Engineering XV(1) (1993), 1-20.
27] M.A. Savi and A.M.B. Braga, Chaotic Response of a Shape Memory Oscillator with Internal Constraints, Proceedings of XII the Brazilian Congress of Mechanical Engineering (COBEM 93 - ABCM), Brasília, Brazil, 1993, 33-36.

[28] M.A. Savi and P.M.L.C. Pacheco, Chaos and Hyperchaos in Shape Memory Systems, International Journal of Bifurcation and Chaos 12(3) (2002), 645-657.

[29] M.A. Savi, P.M.L.C. Pacheco and A.M.B. Braga, Chaos in a Shape Memory Two-Bar Truss, International Journal of Nonlinear Mechanics 37(8) (2002), 1387-1395.

[30] M.A. Savi, A. Paiva, A.P. Baêta-Neves and P.M.C.L. Pacheco, Phenomenological Modeling and Numerical Simulation of Shape Memory Alloys: A Thermo-Plastic-Phase Transformation Coupled Model, Journal of Intelligent Materials Systems and Structures 13(5) (2002), 261-273.

[31] S. Seelecke, Modeling the Dynamic Behavior of Shape Memory Alloys, International Journal of Non-linear Mechanics 37(8) (2002), 1363-1374.

[32] J.M.T. Thompsom and H.B. Stewart, Nonlinear Dynamics and Chaos, John Wiley \& Sons, 1986.

[33] J. van Humbeeck, Non-medical Applications of Shape Memory Alloys, Materials Science and Engineering A 273-275 (1999), 134-148.

[34] R.J. Wasilevski, On the Nature of the Martensitic Transformation, Metalurgical Transactions 6A (1975), 1405-1418.

[35] G. Webb, L. Wilson, D.C. Lagoudas and O. Rediniotis, Adaptive Control of Shape Memory Alloy Actuators for Underwater Biomimetic Applications, AIAA Journal 38(2) (2000), 325-334.

[36] A. Wolf, J.B. Swift, H.L. Swinney and J.A. Vastano, Determining Lyapunov Exponents from a Times Series, Physica $D$ 16 (1985), 285-317. 

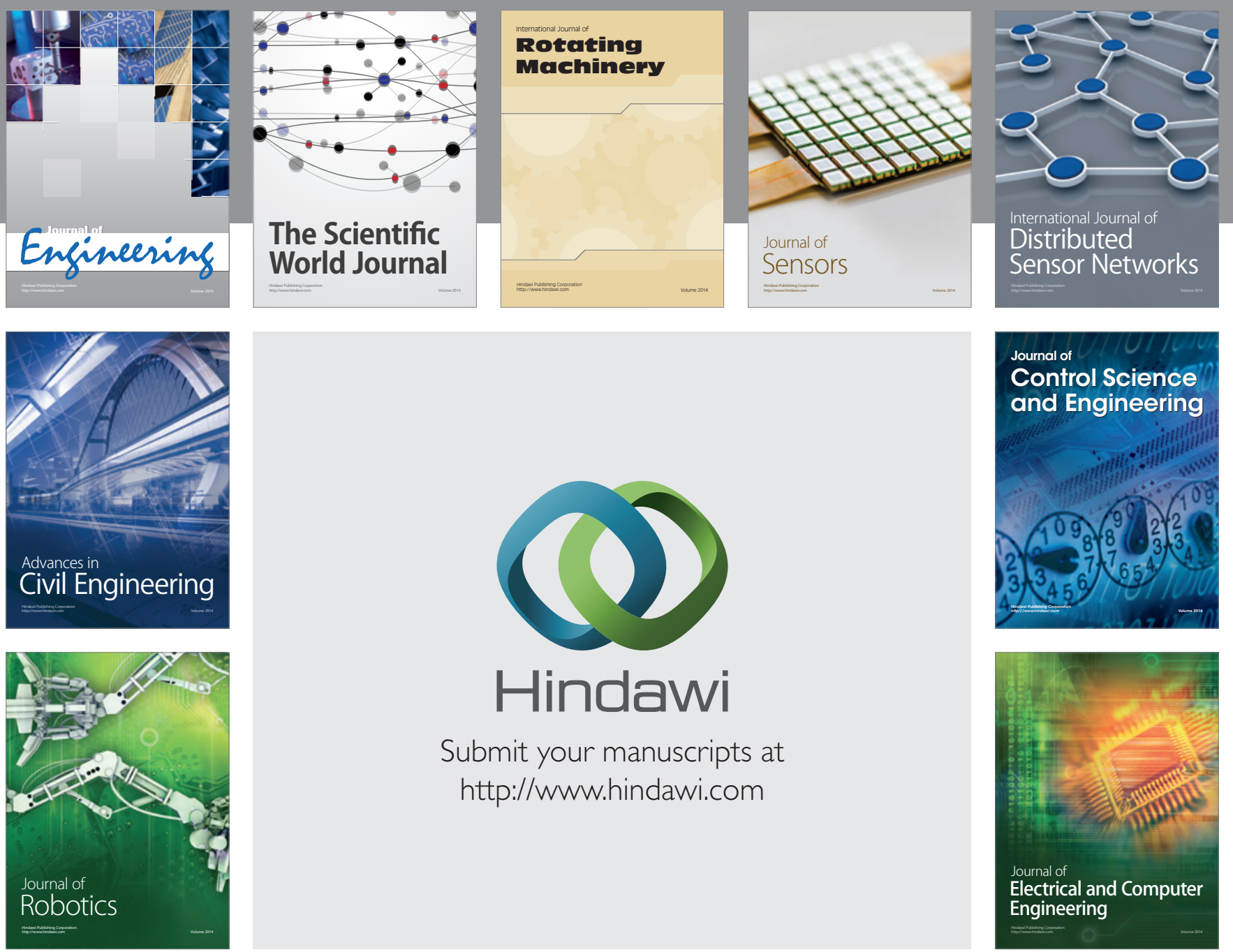

Submit your manuscripts at

http://www.hindawi.com
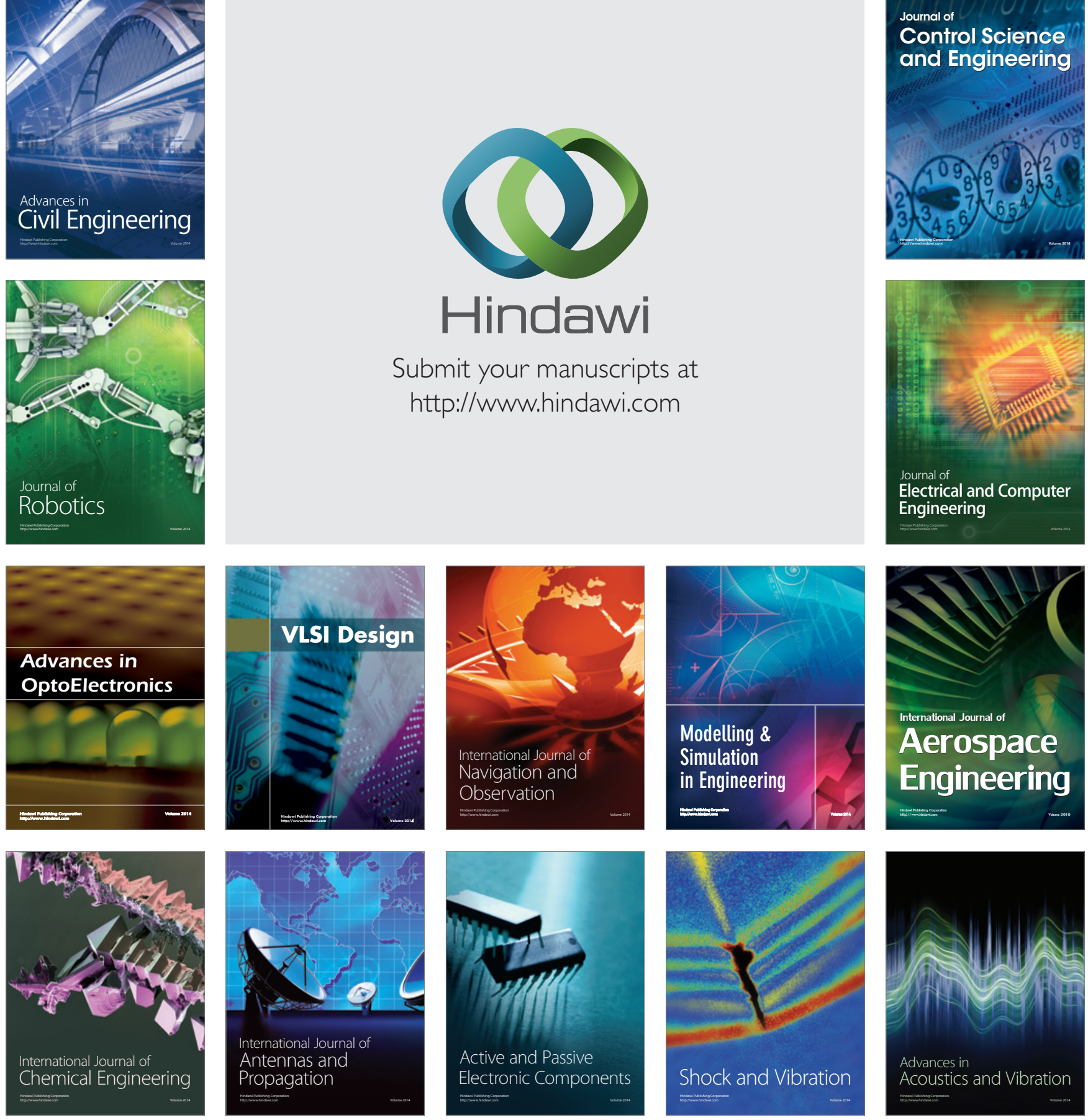